Vol. 22, $n^{\circ} 1 \mid 2018$

Varia

\title{
Gérard Guyon, La justice en questions. Recueils d'articles
}

Limoges, Presses universitaires de Limoges, Cahiers de l'Institut d'anthropologie historique $\mathrm{n}^{\circ} 40,2015,432$ p., $32 €$, ISBN :

978-284287-645-6

\section{Bruno Lemesle}

\section{(2) OpenEdition}

\section{Journals}

Édition électronique

URL : http://journals.openedition.org/chs/2195

DOI : $10.4000 /$ chs. 2195

ISSN : 1663-4837

Éditeur

Librairie Droz

Édition imprimée

Date de publication : 31 octobre 2018

Pagination : 141-143

ISSN : 1422-0857

\section{Référence électronique}

Bruno Lemesle, "Gérard Guyon, La justice en questions. Recueils d'articles », Crime, Histoire \& Sociétés / Crime, History \& Societies [En ligne], Vol. 22, n 1 | 2018, mis en ligne le 31 décembre 2018, consulté le 14 janvier 2021. URL : http://journals.openedition.org/chs/2195; DOI : https://doi.org/10.4000/chs. 2195

Ce document a été généré automatiquement le 14 janvier 2021.

(c) Droz 


\title{
Gérard Guyon, La justice en questions. Recueils d'articles
}

\author{
Limoges, Presses universitaires de Limoges, Cahiers de l'Institut \\ d'anthropologie historique $\mathrm{n}^{\circ} 40,2015,432$ p., $32 €$, ISBN : \\ 978-284287-645-6
}

\section{Bruno Lemesle}

\section{RÉFÉRENCE}

Gérard Guyon, La justice en questions. Recueils d'articles, Limoges, Presses universitaires de Limoges, Cahiers de l'Institut d'anthropologie historique $n^{\circ} 40,2015,432$ p., $32 €$, ISBN : 978-284287-645-6

1 L'ouvrage de Gérard Guyon, qui enseigna l'histoire des institutions et des idées politiques à l'université Montesquieu de Bordeaux, est un recueil de seize articles dont la parution initiale s'est s'échelonnée de 1985 à 2012. Il est lui-même paru l'année précédant le décès de l'auteur, survenu en 2016. Le fil conducteur qui relie les chapitres prolonge une idée séminale de Gabriel Le Bras : il est guidé par le principe qui consiste à ne jamais séparer le domaine juridique de celui de la religion puisque, en France particulièrement (cadre de plusieurs des études du livre), les institutions et la pensée juridique ont été profondément innervées par les institutions chrétiennes. G. Guyon affirmait ainsi qu'il n'avait pas voulu seulement être un historien du droit mais qu'il concevait sa discipline comme devant être largement ouverte à d'autres champs disciplinaires. Ainsi l'auteur a pu prendre la bonne hauteur en faisant de ce livre une somme de petits essais; lorsque la focale s'élargit, même si les évocations sont "superficielles », comme il l'écrit lui-même (p.355), elles ont le mérite de conduire «l'esprit à faire d'utiles comparaisons ». Ajoutons qu'il s'agit d'essais engagés, le plus souvent. Car l'autre trait des textes de G. Guyon est qu'ils manifestent son adhésion affirmée à la religion catholique, adhésion qui guide son regard et davantage encore ses questionnements. Les thèmes traités sont divers : parmi ceux-ci, citons la justice et la 
miséricorde dans la Règle de saint Benoît, l'utopie, la prison, la torture et les clercs, la victime propitiatoire, la justice pénale dans le théâtre religieux médiéval, la violence et le droit... Plutôt que tenter de résumer la teneur de chacun des chapitres, j'évoquerai ceux qui offrent des thèmes récurrents, manifestement chers à l'auteur. L'ouvrage s'ouvre par un chapitre dans lequel il développe un thème dont il avait exposé la teneur dans un article paru en 2003 dans les Cuadernos de Historia del Derecho : intitulé alors « un grand juriste européen : saint Benoît de Nursie ", G. Guyon faisait ressortir, grâce à ce titre volontairement provocateur, un aspect encore méconnu de la Règle de saint Benoît. Dans le présent recueil, il pose la question : «Saint Benoît peut-il être considéré par les juristes comme un des leurs?» et sa réponse, dans l'ensemble positive, se montre nuancée. Car objectif final de la Règle est avant tout « la mise en place de peines médicinales et l'aménagement de rythmes de sanctions efficaces ». Si le vocabulaire de la Règle a souvent une apparence juridique, la réalité qu'elle sert ne relève pas du droit, précisait l'auteur. G. Guyon, sans reprendre tout ce que l'érudition a permis d'établir, mettait l'accent sur l'héritage de saint Benoît qui se situe à la confluence de celui du droit romain et de celui des Pères de l'Église. La grande originalité de la Règle est de contenir un véritable droit de la culpabilité pénale qui tranche complètement avec toutes les pénalités contenues dans les règles antérieures. L'auteur considérait que la législation bénédictine est tout entière ordonnée à la vie spirituelle. Le lecteur trouvera donc exposées des observations désormais bien connues de ceux qui se sont intéressés à ces aspects des règles monastiques anciennes, en particulier des lecteurs de Michel Foucault. La pédagogie de la Règle en matière de culpabilité consiste à provoquer en toutes occasions l'aveu volontaire, mais la faute ne reste pas celle de l'individu, même quand il l'a avouée : elle est prise en charge grâce à une participation active de toute la communauté monastique. En revanche, la pédagogie de la faute est individuelle puisqu'elle met en relation le coupable et l'abbé, faisant de celui-ci à la fois un juge et un pasteur, ce qui souligne le lien étroit qui relie l'abbé et le Christ dont il est l'image vivante dans le monastère. Certains articles de la Règle forment un véritable code pénal. G. Guyon avait noté la minutie avec laquelle sont créées les conditions d'une éducation à la culpabilité, mais il avait curieusement peu développé cet aspect; il n'avait pas mis en valeur l'une des originalités fortes de la Règle par rapport à la pénalité contenue dans le droit romain: la pénalité bénédictine s'attache en effet à toutes les sortes de manquements, de négligences ou d'inobservations : en ce sens, on peut dire qu'elle a aussi pour fonction d'éduquer au respect des normes de conduite, ce que Foucault appelait une « infra-pénalité ». Saint Benoît place la fonction corrective et curative au centre du dispositif de la culpabilité, souligne l'auteur. Ce n'est pas seulement la pédagogie de la correction qui est novatrice mais le processus judiciaire lui-même, en l'occurrence la procédure suivie que l'auteur décrit en signalant qu'elle n'inclut pas l'enquête et en soulignant qu'elle est ordonnée à l'obtention de l'aveu. Toutefois, le long cheminement procédural ne s'adresse pas à ceux qui, en raison de leur nature fruste ou orgueilleuse, sont incapables de se corriger eux-mêmes. À ceux-là la Règle réserve les châtiments corporels, car c'est la nature du délinquant qui requiert le traitement pénal, non celle de la faute. Le juge abbé doit accorder une attention soutenue, presque inquiète, à l'intention coupable. Parmi les autres thèmes, celui de l'utopie a retenu G. Guyon dans plusieurs chapitres du livre. N'écrivait-il pas que «le procès pénal s'organise autour d'une vision juridique idéale d'ordre utopique »? L'utopie comme imaginaire politique dans les différentes formes d'utopies littéraires est pourtant, mais seulement en apparence, un univers sans contraintes légales. Elle est 
une société absolue, un univers statique et cette immobilité est liée à la peur car le réel doit correspondre à un avenir parfait. Le principal souci de l'utopie, de nature pathologique, est la concorde absolue. Totalité absolue, l'utopie n'a pas de territoire pour la prison; si le bannissement reste possible, il ne saurait exister sans risque. Seule subsiste l'intégration totale et (ou) l'élimination radicale. L'étude de l'utopie chez G. Guyon n'était pas un simple intérêt d'ordre intellectuel. Elle traduit bien au contraire une inquiétude profonde dont témoigne le dernier chapitre. C'est l'incarcération qui lui semblait être le principe le mieux relié à l'utopie. La prison est une totalité, elle est " tantôt utopie pratiquée, dans l'idéal religieux vécu des communautés monastiques, tantôt utopie simplement projetée comme un pouvoir dont l'empreinte sur l'ensemble du corps politique et social et le succès dépendent de la force de l'appareil religieux dans l'histoire ou de l'accaparement de son idéologie par l'autorité politique » (p. 75). Il comparait l'incarcération bénédictine à l'incarcération contemporaine, notant que dans la première, la privation de communication signifie que l'exclusion demeure une nécessité mais qu'elle devait se faire à l'intérieur de la communauté. Elle impliquait la réintégration grâce à l'accompagnement des délinquants accompli par des membres de la communauté. Dans le dernier chapitre, consacré à "l'utopie pénale " de la Révolution française, l'auteur affirme que si celle-ci assigne à la peine de prison une fonction d'amendement et de réinsertion sociale, cela n'est pas dû aux thèses des philosophes français du XVIII e siècle. C'est dans la conception de l'Église de la prison que se vérifie le mieux le poids de l'héritage historique sur la nouvelle pénologie de la Révolution. Cependant les ambitions humanitaires du code pénal de 1791 sont plus grandes, comparées à la situation antérieure. Mais l'échec de ce modèle carcéral est patent, entre autres raisons parce que les critère économiques de fonctionnement finissent par l'emporter sur les principes, aussi parce que l'homme est devenu un type de plus en plus abstrait, écrivait G. Guyon ; il est réduit totalement à l'individu. Le livre se conclut alors sur cette interrogation à la fois inquiète et pessimiste: "N'est-il pas possible de montrer que la Révolution française et son utopie carcérale font l'aveu d'une réduction dramatique de l'homme à son utilité politique, sociale et finalement économique?».

\section{AUTEURS}

\section{BRUNO LEMESLE}

Université de Dijon

brunolemesle[at]cegetel.net 